\title{
The Evolution of Resolved Kinematics and Metallicity from Redshift 2.7 to 0.7 with LUCI, SINS/zC-SINF and KMOS ${ }^{3 D}$
}

\author{
Eva Wuyts and the SINS/zC-SINF and KMOS ${ }^{3 \mathrm{D}}$ Teams $^{\ddagger}$ \\ Max-Planck-Institut für extraterrestrische Physik, \\ Giessenbachstr. 1, D-85741 Garching, Germany \\ email: evawuyts@mpe.mpg.de
}

\begin{abstract}
The KMOS ${ }^{3 \mathrm{D}}$ survey will provide near-IR IFU observations of a mass-selected sample of $\sim 600$ star-forming galaxies at $0.7<z<2.7$ with the K-band Multi Object Spectrograph (KMOS) at the VLT. We present kinematic results for a first sample of $\sim 200$ galaxies, focusing on the evolution of the gas velocity dispersion with redshift. Combined with existing measurements, we find an approximate $(1+z)$ evolution from $z \sim 4$ to the present day, which can be understood from the co-evolution of the gas fraction and specific star formation rate (sSFR) in the the equilibrium picture of galaxy evolution.

We combine the $\mathrm{KMOS}^{3 D}$ sample with data from the LUCI and SINFONI spectrographs, as well as multi-wavelength HST imaging from CANDELS, to address the relations between stellar mass, SFR, and the $[\mathrm{N} \mathrm{II}] / \mathrm{H} \alpha$ flux ratio as an indicator of gas-phase metallicity for a sample of 222 star-forming galaxies. We find a constant slope at the low-mass end of the mass-metallicity relation and can fully describe its redshift evolution through the evolution of the characteristic turnover mass where the relation begins to flatten at the asymptotic metallicity. At a fixed mass and redshift, our data do not show a correlation between the $[\mathrm{N} \mathrm{II}] / \mathrm{H} \alpha$ ratio and SFR.
\end{abstract}

Keywords. galaxies: formation - galaxies: evolution - galaxies: high-redshift

\section{Introduction}

The KMOS ${ }^{3 D}$ GTO survey (P. I.: N. M. Förster Schreiber/D. J. Wilman) takes advantage of the multiplex capabilities of the KMOS deployable IFU system at the VLT to observe the $\mathrm{H} \alpha$ and $[\mathrm{N} \mathrm{II}]$ emission of a mass-selected sample of $\sim 600$ star-forming galaxies (SFGs) at $0.7<z<2.7$ in the COSMOS, GOODS-South and UDS deep fields. Targets are selected from the rest-frame optical 3D-HST Treasury Survey (Brammer et al. 2012), which reduces the historical bias of previous rest-frame UV-selected IFU studies towards blue star-forming galaxies. The crucial synergy of KMOS ${ }^{3 \mathrm{D}}$ with the CANDELS multi-wavelength HST imaging allows to connect the properties of the ionised gas (kinematics, SF, metallicity and outflows) with the stellar structure and populations, as well as galaxy environment.

$\ddagger$ Jaron Kurk ${ }^{1}$, Natascha M. Förster Schreiber $^{1}$, Reinhard Genzel ${ }^{1}$, Emily Wisnioski ${ }^{1}$, Kaushala Bandara ${ }^{1}$, Stijn Wuyts $^{1}$, Alessandra Beifiori ${ }^{1}$, Ralf Bender ${ }^{1}$, Gabriel B. Brammer, Andreas Burkert, Peter Buschkamp ${ }^{1}$, C. Marcella Carollo, Jeffrey Chan ${ }^{1}$, Ric Davies ${ }^{1}$, Frank Eisenhauer $^{1}$, Max Fabricius, Matteo Fossati ${ }^{1}$, Sandesh K. Kulkarni ${ }^{1}$, Yoshi Fudamoto ${ }^{1}$, Philipp Lang $^{1}$, Simon J. Lilly, Dieter Lutz ${ }^{1}$, Chiara Mancini, Trevor Mendel ${ }^{1}$, Ivelina G. Momcheva, Thorsten Naab, Erica J. Nelson, Alvio Renzini, David Rosario ${ }^{1}$, Roberto P. Saglia ${ }^{1}$, Stella Seitz, Ray M. Sharples, Amiel Sternberg, Sandro Tacchella, Linda J. Tacconi ${ }^{1}$, Pieter van Dokkum, David J. Wilman ${ }^{1}$ 


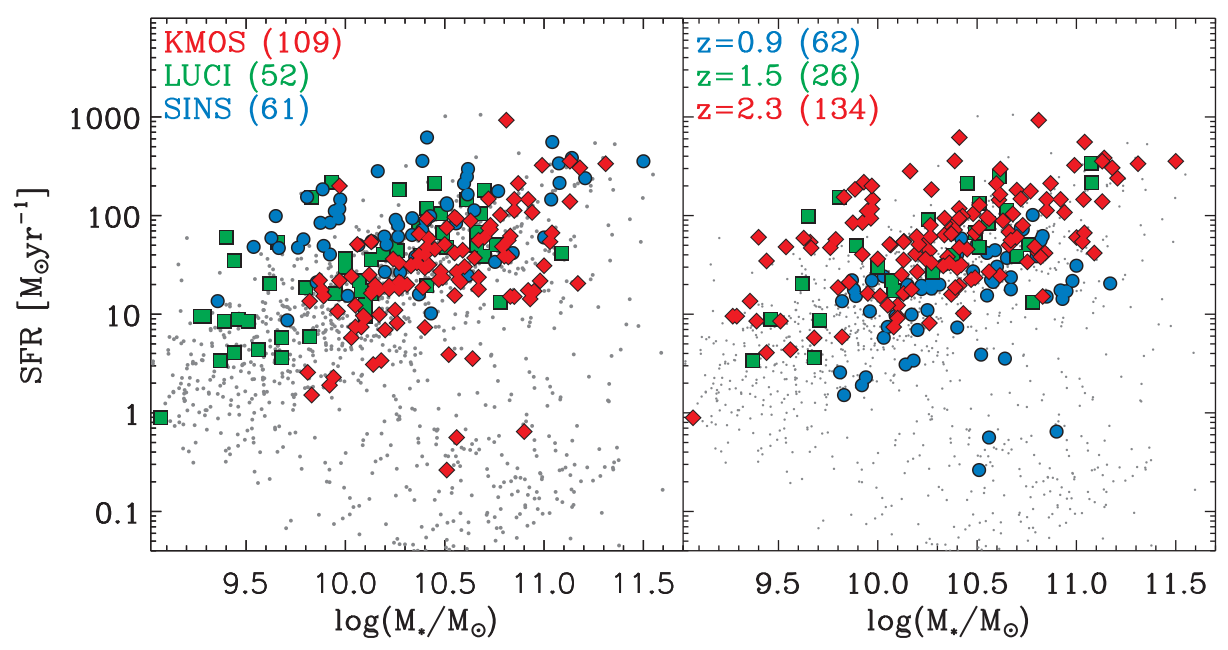

Figure 1. Location of the combined $\mathrm{KMOS}^{3 \mathrm{D}}$, LUCI and SINS sample in the SFR-stellar mass plane. Targets are color-coded by instrument in the left panel and redshift in the right panel. The numbers in parentheses note the number of sources in each survey or redshift bin. As a reference, the small gray dots represent the total mass-selected galaxy population at $0.7<z<2.7$ in the CANDELS/3D-HST fields.

\section{The redshift evolution of the velocity dispersion}

The first year of $\mathrm{KMOS}^{3 \mathrm{D}}$ observations has targeted 223 SFGs, 106 galaxies in $Y J$ band at median $z=0.9$ and 117 galaxies in $K$-band at median $z=2.3$ (Figure 1 ), and has detected $\mathrm{H} \alpha$ emission for $80 \%$ of them. For all detected targets, we have carried out the standard kinematic extraction to produce maps of $\mathrm{H} \alpha$ flux, velocity and velocity dispersion as well as $1 \mathrm{D}$ velocity and dispersion profiles along the kinematic axis. When these 1D profiles are well-defined, we fit an exponential disk model and measure the dispersion in the outer regions of the galaxy, at or outside the turnover radius, to minimize the impact of beam-smearing in the inner regions. We find an average dispersion of $50 \mathrm{~km} / \mathrm{s}$ at $z=2.3$ and $25 \mathrm{~km} / \mathrm{s}$ at $z=0.9$ (Wisnioski et al. 2014, in prep.). Figure 2 combines the $\mathrm{KMOS}^{3 \mathrm{D}}$ dispersion results with literature data for a range of different surveys and shows a continuous $(1+z)$ decline in dispersion from $z \sim 4$ towards the present day. This can be physically understood starting from the Toomre stability criterium, where for a critical disk $\sigma(z) \sim v_{\text {rot }} f_{\text {gas }}(z)$ (Genzel et al. 2008). The redshift evolution of the gas fraction $f_{\text {gas }}(z)$ depends on the evolution of the depletion time and specific star formation rate. Adopting recent scaling relations from the literature (Whitaker et al. 2014; Tacconi et al. 2013; Genzel et al. 2014a), the shaded gray area in Figure 2 shows the predicted velocity dispersion evolution for a range of rotational velocities $v_{\text {rot }}=100-250 \mathrm{~km} / \mathrm{s}$ (see Wisnioski et al. 2014 (in prep.) for more details). The agreement with the data supports the equilibrium picture of galaxy evolution, where the increased turbulence in high redshift disks is driven by their higher gas fractions.

\section{The redshift evolution of the mass-metallicity relation}

We measure the average $[\mathrm{N} \mathrm{II}] / \mathrm{H} \alpha$ line ratio for all $\mathrm{KMOS}^{3 \mathrm{D}}$ targets from an integrated, velocity-shifted $1 \mathrm{D}$ spectrum within the maximal elliptical aperture along the kinematic axis. To increase the statistics and redshift coverage of this metallicity study, we include existing LUCI long-slit and SINFONI IFU spectroscopic data (Förster Schreiber et al. 2009; Mancini et al. 2011), adding 26 SFGs at $z \sim 1.5$ and 87 SFGs at $z \sim 2.3$ 

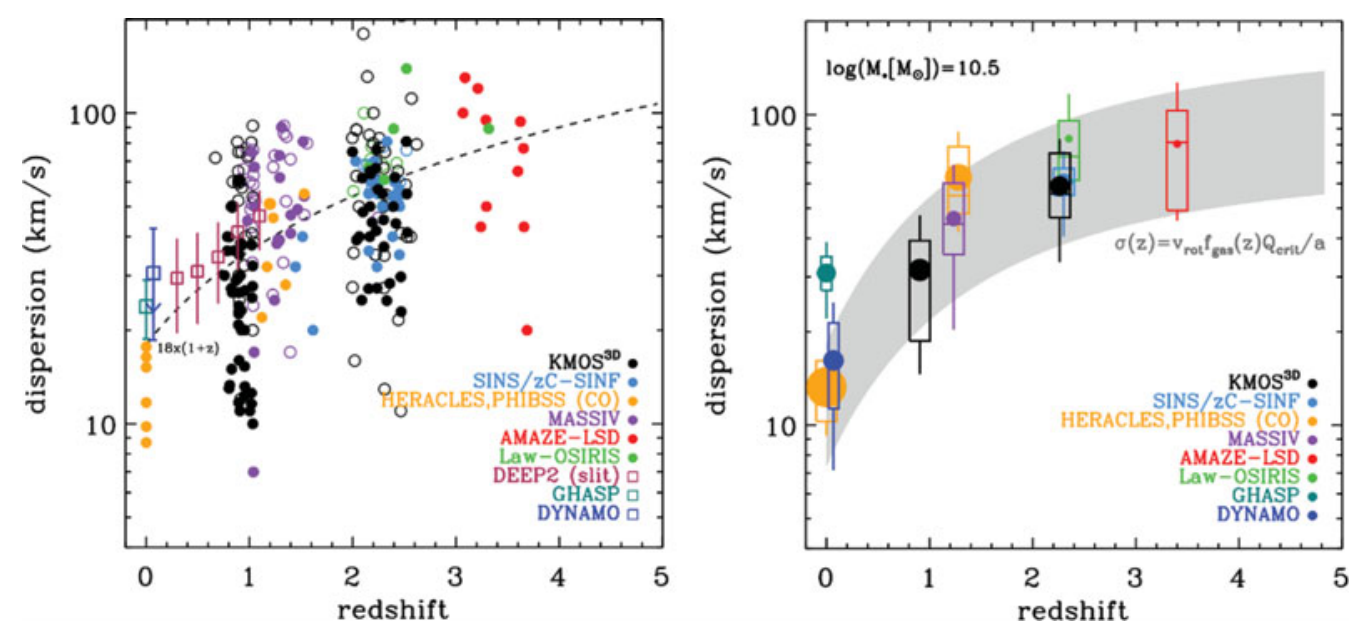

Figure 2. Left: Galaxy velocity dispersion measurements from the literature at $z=0-4$ from molecular and ionized gas emission. $\mathrm{KMOS}^{3 \mathrm{D}}$ results at $z=0.9$ and $z=2.3$ are shown by black circles. The dashed line shows a simple $(1+z)$ evolution scaled to overlap with the data. Right: The gray band shows the expected dispersion based on the Toomre criterium and the redshift evolution of the gas fraction (see text). It agrees well with the mean (filled circles) and $50 \%$ and $90 \%$ distributions (boxes and vertical lines, respectively) of the data points shown on the left.

(Figure 1). Our analysis is carried out as much as possible in terms of the directly observable $[\mathrm{N} \mathrm{II}] / \mathrm{H} \alpha$ line ratio to avoid systematics associated with the choice of strong-line metallicity calibration (Kewley \& Ellison 2008); when necessary, we employ the linear calibration by Pettini \& Pagel (2004).

Figure 3 shows the $[\mathrm{N} \mathrm{II}] / \mathrm{H} \alpha$ ratios as a function of stellar mass, $2 \sigma$ upper limits are included for the $17 \%$ of targets for which [N II] was not detected. We identify 18 AGNs in our sample from X-ray and radio data, infrared colors, and rest-UV spectroscopy (Genzel et al. 2014a). Additionally, Förster Schreiber et al. (2014) and Genzel et al. (2014a) report evidence for AGN-driven outflows in the nuclear regions of 20 targets included in our combined sample from broad (FWHM $\gtrsim 1000 \mathrm{~km} / \mathrm{s}$ ) emission components with enhanced $[\mathrm{N} \mathrm{II}] / \mathrm{H} \alpha$ line ratios. We stack our galaxies at $z=0.9$ and $z=2.3$, both with and without the AGN-contaminated sources. The right panel of Figure 3 shows that the $17 \%$ AGN contamination of our sample does not significantly affect the derived mass-metallicity relation (MZR). We find good agreement with literature studies at the high mass end (Erb et al. 2006; Zahid et al. 2014b; Steidel et al. 2014). The different slopes at the low-mass end are likely due to differences in sample selection, especially the inclusion of red, dusty objects. This needs further investigation.

We provide fits to the MZR based on the parameterization introduced by Zahid et al. (2014a),

$$
12+\log (\mathrm{O} / \mathrm{H})=Z_{0}+\log \left[1-\exp \left(-\left[\frac{M_{*}}{M_{0}}\right]^{\gamma}\right)\right]
$$

where $Z_{0}$ corresponds to the asymptotic metallicity, $M_{0}$ is the characteristic turnover mass where the relation begins to flatten and $\gamma$ is the power-law slope at stellar masses $\ll M_{0}$. Within the uncertainties, $Z_{0}$ and $\gamma$ are constant, such that redshift evolution of the MZR depends solely on the evolution of the characteristic turnover mass (Wuyts et al. 2014). For a fixed $Z_{0}=8.69$ and $\gamma=0.4$, we can describe the redshift evolution of the characteristic turnover mass as $\log \left(M_{0} / \mathrm{M}_{\odot}\right)=(8.86 \pm 0.05)+(2.92 \pm 0.16) \log (1+z)$. The 


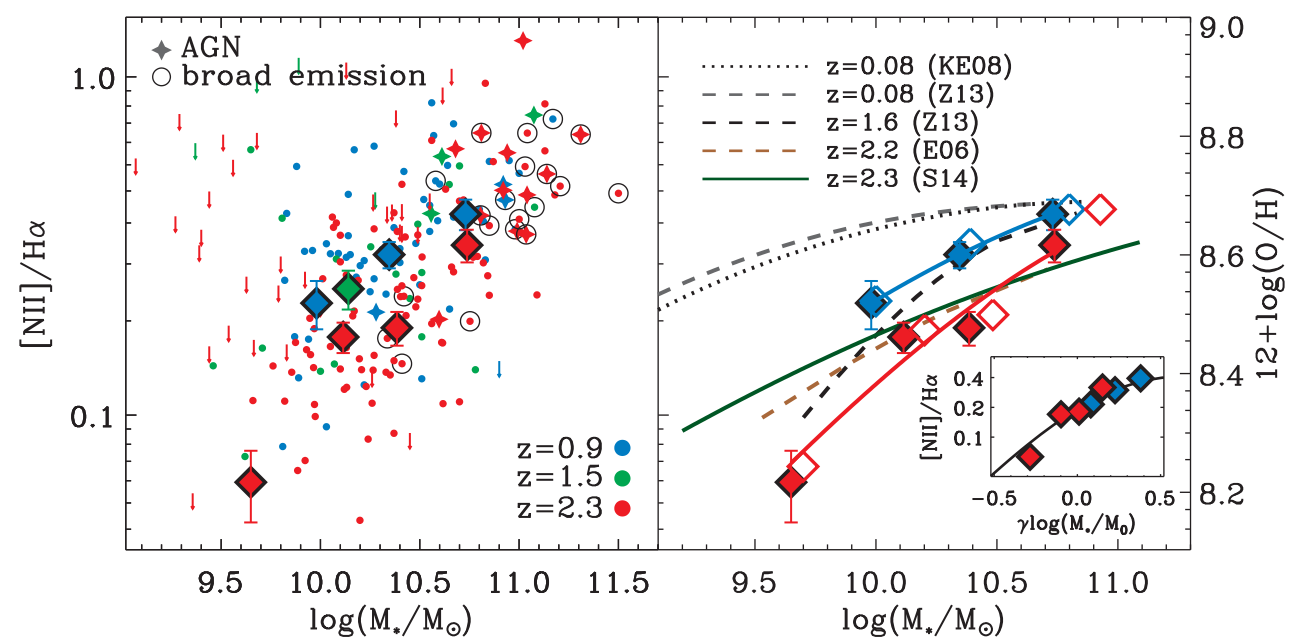

Figure 3. $[\mathrm{N} \mathrm{II}] / \mathrm{H} \alpha$ detections and $2 \sigma$ upper limits vs. stellar mass, color-coded by redshift. AGNs identified from classic X-ray, etc., indicators and broad nuclear AGN-driven outflows are indicated with four-pointed stars and black circles, respectively. Large colored diamonds indicate the stacked $[\mathrm{N} \mathrm{II}] / \mathrm{H} \alpha$ ratios in three, one, and four bins of stellar mass for the $z=0.9, z=1.5$, and $z=2.3$ redshift slices, excluding the AGN-contaminated sources. In the right panel, the open diamonds show the stacked results when all galaxies are included. The blue and red solid lines correspond to our best-fit MZRs. The inset shows the stacked $[\mathrm{N} \mathrm{II}] / \mathrm{H} \alpha$ ratios as a function of $\gamma \log \left(M_{*} / M_{0}\right)$, which follow a universal relation.

relation between metallicity and stellar mass scaled by $M_{0}$ thus becomes independent of redshift, as can be seen in the right panel of Figure 3.

Finally, we investigate the importance of SFR in the mass-metallicity relation by stacking our sample at $z=0.9$ and $z=2.3$ in two bins of SFR for each mass bin. We do not find a correlation between the $[\mathrm{N} \mathrm{II}] / \mathrm{H} \alpha$ ratio and SFR at fixed mass and redshift (Wuyts et al. 2014). Both metallicity and SFR evolve with redshift, but the lack of correlation between these two parameters at fixed mass and redshift, suggests that both processes are not necessarily causally related.

\section{References}

Brammer, G. B., et al. 2012, ApJS, 200, 13

Erb, D. K., et al. 2006, ApJ, 644, 813

Förster Schreiber, N. M., et al. 2009, ApJ, 706, 1364

Förster Schreiber, N. M., et al. 2014, ApJ, 787, 38

Genzel, R., et al. 2008, ApJ, 687, 59

Genzel, R., et al. 2014a, arXiv:1406.0183

Genzel, R., et al. 2014b, arXiv:1409.1171

Kewley, L. J. \& Ellison, S. L. 2008, ApJ, 681, 1183

Mancini, C., et al. 2011, ApJ, 743, 86

Pettini, M. \& Pagel, B. E. J. 2004, MNRAS, 348, L59

Steidel, C. C., et al. 2014, arXiv:1405.5473

Tacconi, L. J., et al. 2013, ApJ, 768, 74

Whitaker, K. E., et al. 2014, arXiv:1407.1843

Wuyts, E., et al. 2014, ApJ, 789, L40

Zahid, H. J., et al. 2014a, ApJ, 791, 130

Zahid, H. J., et al. 2014b, ApJ, 792, 75 